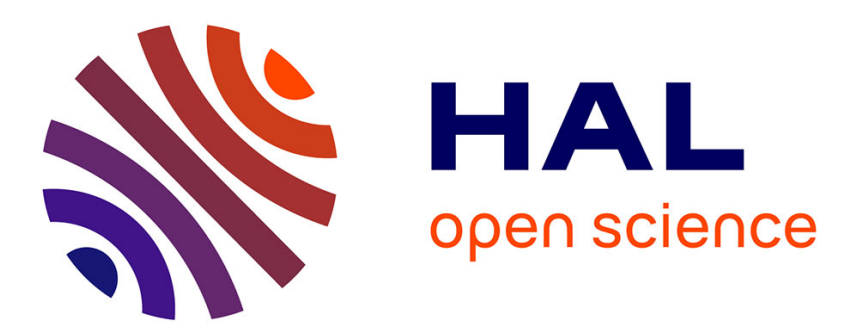

\title{
Drying-induced bending deformation of cellulose nanocrystals studied by molecular dynamics simulations
}

Yu Ogawa, Yoshiharu Nishiyama, Karim Mazeau

\section{To cite this version:}

Yu Ogawa, Yoshiharu Nishiyama, Karim Mazeau. Drying-induced bending deformation of cellulose nanocrystals studied by molecular dynamics simulations. Cellulose, 2020, 27 (17), pp.9779-9786. 10.1007/s10570-020-03451-9 . hal-02951214

\section{HAL Id: hal-02951214 \\ https://hal.science/hal-02951214}

Submitted on 1 Dec 2020

HAL is a multi-disciplinary open access archive for the deposit and dissemination of scientific research documents, whether they are published or not. The documents may come from teaching and research institutions in France or abroad, or from public or private research centers.
L'archive ouverte pluridisciplinaire HAL, est destinée au dépôt et à la diffusion de documents scientifiques de niveau recherche, publiés ou non, émanant des établissements d'enseignement et de recherche français ou étrangers, des laboratoires publics ou privés. 


\section{Drying-induced bending deformation of cellulose nanocrystals studied by}

\section{2 molecular dynamics simulations}

3 Yu Ogawa, ${ }^{*}$ Yoshiharu Nishiyama, ${ }^{*}$ Karim Mazeau

4 Univ. Grenoble Alpes, CNRS, CERMAV, 38000, Grenoble, France

$5 \quad *$ Corresponding authors: (Y.O.) yu.ogawa@cermav.cnrs.fr

ORCID: (Y.O.) 0000-0003-0677-7913 (Y.N.) 0000-0003-4069-2307

8

\section{ABSTRACT}

10 Drying cellulosic materials from their water-swollen state can collapse their ultrastructure and alter their macroscopic material properties such as mechanical strength and waterretention ability. However, at the single-crystal or molecular level, little is known about the deformation of cellulose upon drying. We thus investigate herein the drying-induced deformation of a cellulose crystal by using an atomistic molecular dynamics simulation that considers a hydrated system composed of two short cellulose crystals, a lower one fixed to a flat substrate and an upper one free to deform. To mimic vacuum drying, the water is gradually removed from the system. As the drying proceeds, the upper cellulose crystal bends and forms a tight contact with the lower cellulose crystal. This result underlines the importance of lateral deformation of cellulose crystals in the collapse of the cellulose ultrastructure and provides insights into the molecular mechanisms responsible for modifying the properties of cellulose materials.

23 Keywords: cellulose; drying; lateral deformation; molecular dynamics 
INTRODUCTION

Cellulose is extracted and processed in the aqueous condition in many industrial processes, from chemical pulping to fiber and textile production to nanocellulose production. As a result, producing cellulosic materials often requires drying or water removal. Drying cellulosic materials modifies its physical properties; for instance, in the process of paper manufacturing, pulp fibers stiffen and shrink laterally upon drying (Minor, 1994; Giacomozzi and Joutsimo, 2017). In addition, once dried, the pulp does not fully recover to the original state upon rewetting, and repeated drying and wetting produces a more rigid and brittle material. This phenomenon, called "hornification," poses a challenge to paper recycling because hornified fibers do not reswell to their initial hydrated state when resuspended in water, which weakens recycled paper. Such degradation of the material properties upon conventional drying (air, oven, contact, etc.) occurs not only with pulp fibers but also with different cellulosic materials such as hydrogels and nanocellulose materials (Peng et al., 2012; Rämänen et al., 2012). Retaining the fine structure of cellulosic materials and minimizing the impact of drying often requires more gentle and costly drying methods such as freeze-drying or critical-point drying (Jin et al., 2004; Heath and Thielemans, 2010; Hoepfner et al., 2008; Beaumont et al., 2017). Conversely, simple air drying also produces some exceptional material properties in cellulosic materials. For example, a cast film of cellulose nanofiber dried from an aqueous suspension forms an exceptionally high barrier against oxygen gas (Fukuzumi et al., 2009), which means that this simple drying method packs the cellulose nanofibers tightly enough to prevent gas molecules from permeating through the film.

These drying-induced modifications in the mechanical properties are due to microscopic, irreversible structural changes within cellulosic materials and at various length 
scales (Hult et al., 2003; Newman, 2004; Oksanen et al., 1997). Given that, compared with

the rigidity of cellulose the surface tension of water is very significant at the nanometric scale, the capillary force can collapse the ultrastructures such as micropores and nanofiber networks, as has been observed by using various analytical techniques (e.g., microscopy, nitrogen adsorption, solid-state NMR spectroscopy, differential scanning calorimetry) (Häggkvist et al., 1998; Lovikka et al., 2016; Park et al., 2006). Although this interpretation of the drying effect is well accepted, what remains unclear is how cellulose deforms at the single-crystal and molecular levels in these collapsed structures. Recent reports based on transmission electron microscopy and scanning probe microscopy testify to the increasing interest in the nanoscale deformation of cellulose crystals (Mattos et al., 2019; Ogawa, 2019; Smith et al., 2019). Unfortunately, limitations in characterization methods (e.g., spatial resolution) and the intrinsic heterogeneity of cellulose morphology hinder the understanding of such deformations on a molecular level. More detailed molecular-level descriptions are thus needed to fully understand the deformation mechanism of cellulose crystals and how it affects material properties.

We recently demonstrated that, for cellulose crystals, force-field-based atomistic simulations provide structural insights into various deformation modes such as shear and bending deformations (Chen et al., 2016; 2018; Molnár et al., 2018). These computational studies indicate that crystalline cellulose is highly pliant in its lateral plane, contrary to the general assumption that crystalline cellulose forms rigid rods. In the present study, we use atomistic molecular dynamics (MD) to simulate a simplified system to determine the deformation of cellulose crystals upon drying. The results indicate that crystalline cellulose undergoes a bending deformation upon gradual removal of water from a hydrated system composed of two short cellulose crystals. 


\section{COMPUTATIONAL METHODS}

74

75

3

\section{Simulation setup}

All simulations were done with GROMACS (Abraham et al., 2015) using the GROMOS 56Acarbo force field (Hansen and Huenenberger, 2011), including the optimized LennardJones repulsive parameter for the united $\mathrm{CH} 1$ atom type (Chen et al., 2014). This optimized GROMOS force field has already been used to investigate the lateral deformation of cellulose crystals in vacuum, which is similar to the simulation presented herein (Chen et al., 2016; Molnár et al., 2018). Although not designed specifically to model such a solid-vacuum interface, the deformation of cellulose crystals given by the force field is quantitatively consistent with the results of finite-element analysis and qualitatively consistent with transmission electron microscopy observations of kinked cellulose crystals (Chen et al., 2016). Thus, we believe that it can reproduce the deformation of cellulose crystals induced by dehydration.

MD simulations were done in the NVT ensemble with a 2 fs integration time step. The stochastic velocity-scaling algorithm of Bussi et al. (2007) was used to maintain the temperature at $300 \mathrm{~K}$, and $\mathrm{O}-\mathrm{H}$ bonds were constrained by using the LINCS algorithm (Hess et al., 1997). The long-range electrostatic interactions and Lennard-Jones interactions were both calculated by using the cutoff method with a cutoff distance of $0.9 \mathrm{~nm}$. The analyses were done by using GROMACS and in-house scripts. The MD structures were visualized by using PyMOL (PyMOL ver. 2.1.0).

\section{Model construction}


The cellulose microfibril model of the major native allomorph $1 \beta$ was built from crystal

structures (Nishiyama et al., 2002) by considering the dominant hydrogen-bond network, pattern A. The crystal had dimensions of approximately $3.5 \mathrm{~nm} \times 3.5 \mathrm{~nm} \times 20 \mathrm{~nm}$ and

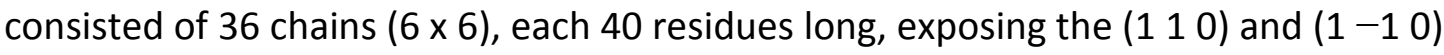
surfaces. Note that the crystal width is similar to that of higher plant cellulose microfibrils $(\approx 3 \mathrm{~nm})$, whereas the crystals are much shorter than natural cellulose microfibrils.

This crystal model was duplicated and two crystals were positioned parallel to each other, separated by $1.8 \mathrm{~nm}$, in a small simulation box $\left(4.5 \times 9.5 \times 22 \mathrm{~nm}^{3}\right)$, as shown in Fig. 1a. The system was then solvated with simple-point-charge water (Berendsen et al., 1981), and the energy was minimized by using the steepest descent method followed by the conjugate gradient method, with the convergence criterion being a maximum force of $10 \mathrm{~kJ} \mathrm{~mol}^{-1} \mathrm{~nm}^{-1}$. The positions of the selected atoms were then constrained as shown in Fig. $1 \mathrm{~b}$ to maintain the separation between the central sections of the crystals, and this position constraint was retained in the following MD simulations. The system was then stepwise heated in the NPT ensemble from 0 to $300 \mathrm{~K}$ in $20 \mathrm{~ns}$. The pressure was regulated by using a Berendsen barostat with a relaxation time of 2.0 ps (Berendsen et al., 1984).

\section{General strategy for modeling the drying process}

To demonstrate the drying-induced deformation of cellulose, we studied the simple model shown in Figure 1. As illustrated in Fig. 1a, we considered a geometry where an upper cellulose crystal is positioned parallel to and above another crystal laid on a flat substrate, and a crystal is sandwiched crossways between the two parallel crystals to maintain a constant separation between them. We used this crossed-fibril geometry to mimic the random orientation of cellulose crystals in a film drop-casted from aqueous suspensions 
119 (Fukuzumi et al., 2009). In the model studied, all atomic positions in the bottom crystal were 120 constrained except those at the upper surface that faced the upper crystal. The third crystal

121 was treated implicitly to reduce the system size. The inter-crystal spacing was maintained by

122 constraining the atomic positions of four residues at the center of each molecular chain on

123 the bottom layer of the upper crystal. The side surfaces of the cellulose crystals were

124 hydrophilic $\left(\begin{array}{lll}1 & -1 & 0\end{array}\right)$ and $\left(\begin{array}{lll}1 & 1 & 0\end{array}\right)$, and the surfaces facing the opposing crystal were both

125 (1 110$)$ surfaces.

126 Water evaporation was modeled by stepwise removal of water molecules in the gas

127 phase. First, the hydrated system was heated to $300 \mathrm{~K}$, and then the volume of the

128 simulation box was expanded fourfold $(10 \times 15 \times 25 \mathrm{~nm})$ to generate vacuum space. The MD

129 simulation was then executed at a constant volume at $300 \mathrm{~K}$ but was stopped every $100 \mathrm{ps}$

130 to remove isolated water molecules ("isolated" water molecules were defined as those with

131 no neighbor molecule within $1 \mathrm{~nm}$ ). Upon restarting the simulation, the atomic velocities

132 from the previous MD trajectory were retained, so the system temperature remained

133 essentially constant at $300 \mathrm{~K}$. 
(a)

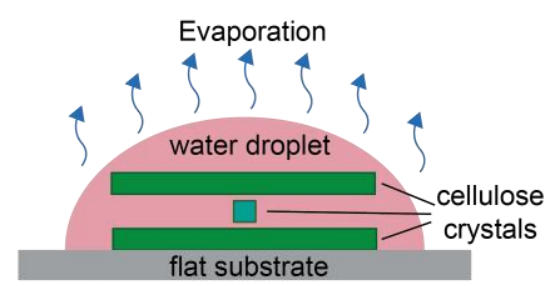

(b)

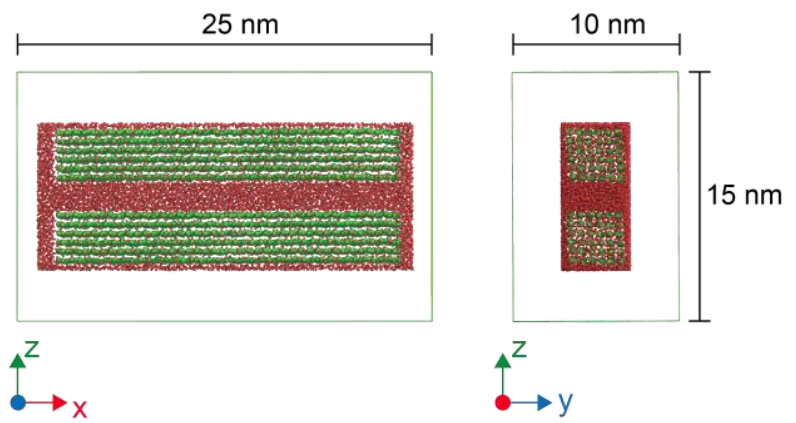

(c)

reducing end non-reducing end

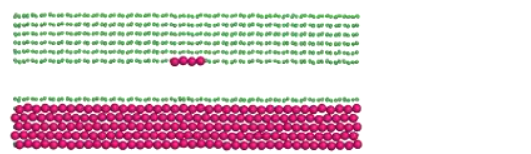

constrained

135 Figure 1. Model used to simulate drying of cellulose crystals: (a) Schematic drawing showing

136 geometry of simulation. (b) Initial dimensions of simulation box containing two crystals and

137 water molecules. For clarity, only the $\mathrm{C} 1$ atoms of cellulose (green) and the oxygen atoms of

138 water (red) appear in these snapshots. (c) Constraints on atomic positions within cellulose

139 crystals. The positions of the magenta glucose residues were constrained in the $x, y$, and $z$

140 directions. For simplicity, only C1 atoms (green) are shown for each residue, but the

141 constraints were applied to all atoms in the residues. The left and right extremities of the

142 upper (lower) surface of the lower (upper) cellulose crystal are called the reducing and non-

143 reducing ends, respectively. The cellulose crystals are maintained in this orientation in all

144 figures presented herein. 
Figure 2 summarizes the general features of the drying process. As shown in Fig. $2 a$, the

148 number of water molecules decreases monotonically in time, indicating that the strategy

149 adopted is suitable for simulating the gradual removal of water on the nanometer scale.

150 Almost all water molecules are removed after $340 \mathrm{~ns}$ (32 and 8 water molecules remain at

151340 and $350 \mathrm{~ns}$, respectively). Figure $2 \mathrm{~b}$ shows snapshots of the simulated system during

152 the drying process and reveals a bending deformation of the upper cellulose crystal and the

153 heterogeneous removal of water.

154

(a)

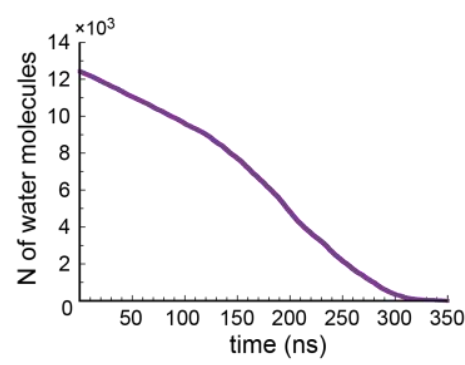

$150 \mathrm{~ns}$

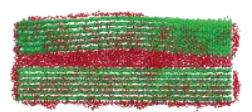

$270 \mathrm{~ns}$

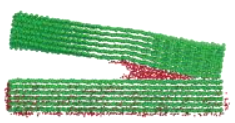

(b)

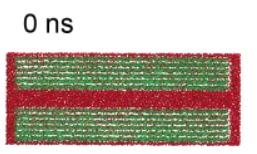

$$
70 \mathrm{~ns}
$$

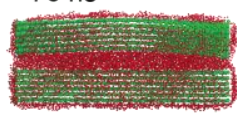

190 ns
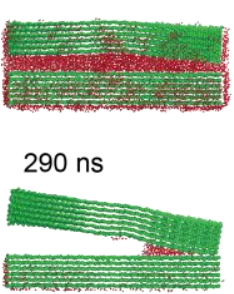

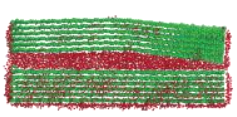

310 ns

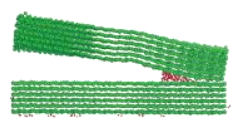

$10 \mathrm{~ns}$

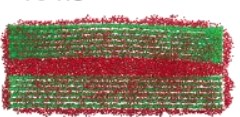

$90 \mathrm{~ns}$

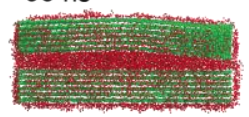

210 ns

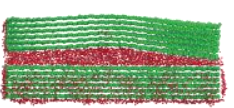

330 ns

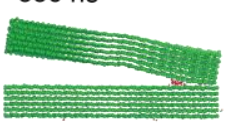

$30 \mathrm{~ns}$

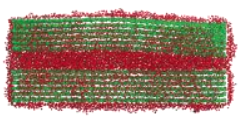

110 ns

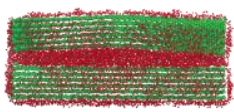

230 ns

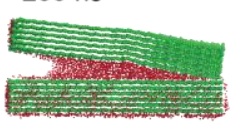

340 ns

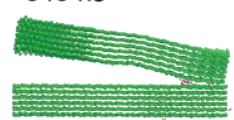

50 ns

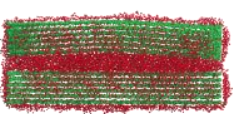

130 ns

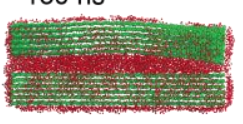

$250 \mathrm{~ns}$

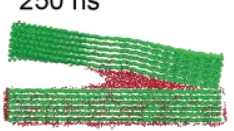

155

Figure 2. Result of drying simulation: (a) Number of water molecules as a function of time.

(b) Snapshots of simulated cellulose-crystal system during drying.

\section{Deformation of cellulose crystal}

At $10 \mathrm{~ns}$, the upper cellulose crystal deforms from its initial linear form into a

162 simulation as can be seen in the bending angle evolution in Fig. 3b. The curved morphology 
indicates that the surface tension of water suffices to cause a lateral deformation of very short cellulose crystals. At this point, the upper cellulose crystal becomes convex with respect to the lower cellulose crystal and roughly symmetric with respect to the center of the crystal (Fig. 3b).

168 The curved morphology persists until about $150 \mathrm{~ns}$, at which point most water molecules have been removed from the upper and side faces of the upper crystal. After

170170 ns the crystal becomes asymmetrically bent about its center. The lower crystal surface

171 at the non-reducing end (right-hand end) approaches to within significantly less than $0.9 \mathrm{~nm}$

172 of the upper surface of the bottom crystal, which is the cutoff distance for non-bonding 173 interactions in this simulation. At 220 ns (Fig. 3), the edge of the non-reducing end of the 174 upper crystal makes contact with the bottom crystal. The bending angle of the upper crystal 175 increases from $4^{\circ}$ in the initial curved state (i.e., at $10 \mathrm{~ns}$ ) to $10^{\circ}$. The reducing end of the upper crystal has moved upward, away from the bottom crystal. This displacement of the

177 reducing end is initiated by the rupture of the continuous water layer that previously forced 178 the curved morphology of the upper crystal. As the drying proceeds further, the crystal179 contact area expands and produces surface-to-surface contact (Figs. 3a and 4b). After 240 ns, this contact area remains constant because the bending angle for the non-reducing end

181 remains constant once the contact area is well established. In contrast, the bending angle of 182 the free reducing end fluctuates (Fig. 3b). The non-reducing end of the upper crystal forms a 183 concave curvature. As detailed below, the water molecules between the two crystals are expelled from the contact area. 
(a)
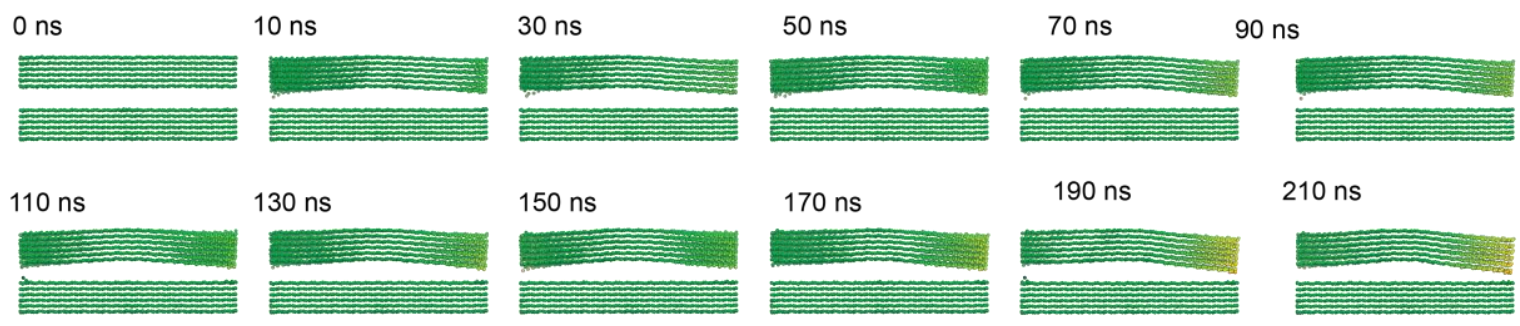

210 ns

230 ns

290 ns

310 ns

330 ns
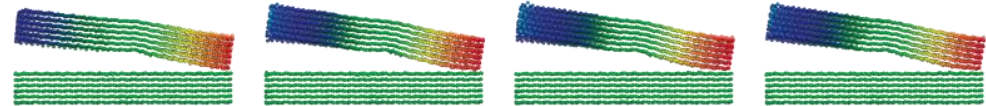

250 ns

270 ns

340 ns
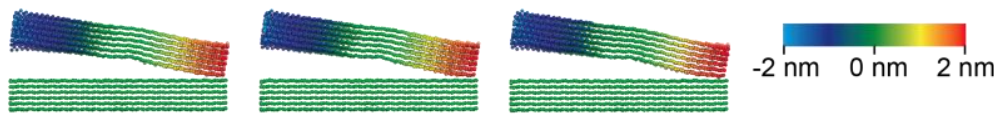

(b)

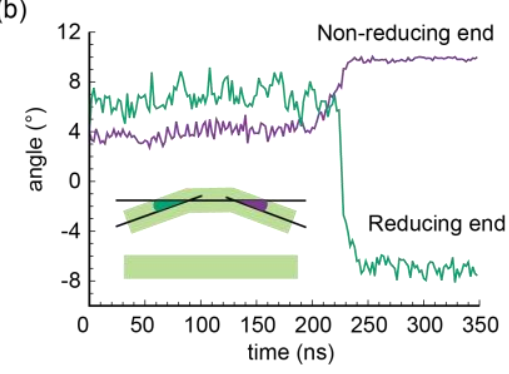

187 Figure 3. (a) Deformation of upper cellulose crystal in drying simulation. The color code

188 indicates the atomic displacement in the $z$ direction (vertical direction in the figure). (b)

189 Bending angle plotted as a function of time during drying for upper cellulose crystal at

190 reducing end (green) and non-reducing end (purple). The bending angles are the angles

191 between the tangent to the central section and the tangents to the ends of the crystal, as

192 schematized in the inset.

193

194

Water removal

195

Immediately after the simulation box expands fourfold in volume, the surface tension of

water causes the water molecules to form an elongated droplet surrounding the two

197

cellulose crystals (Fig. 2). The water molecules gradually evaporate from the outer side of

the droplet, whereas the number of water molecules trapped between the cellulose crystals

remains roughly constant. As mentioned above, most of the water molecules around the

200 crystals have evaporated after $170 \mathrm{~ns}$, leaving only a monolayer of water molecules directly

201 adsorbed on the cellulose-crystal surfaces. As seen in Fig. 2a, the evaporation rate increases 
strong interaction exists between the cellulose-crystal surface and the water. The position constraints that lead to a temperature of $0 \mathrm{~K}$ for the constrained part of the crystal means that water adsorbed on the bottom crystal evaporates much more slowly than water adsorbed on the upper crystal.

Once the contact between the cellulose crystals occurs at $220 \mathrm{~ns}$, a water meniscus forms on the reducing end where the two crystals are widely separated (Fig. 4a). The meniscus is concave, which is indicative of strong adhesion between water and the cellulose-crystal surfaces. As the drying proceeds further, the water molecules evaporated

211 from the reducing end (i.e., the left side where the crystals are separated), and the meniscus surface gradually moves toward the non-reducing end where the cellulose crystals are in contact. The water molecules are retained in this opening until the end of the drying process, as indicated by the few molecules still apparent at 340 ns in Fig. 3.

Conversely, the water molecules near the contact area between the two crystals are expelled independently of the drying within the open space between the reducing ends of

217 the cellulose crystals. Figure $4 \mathrm{~b}$ shows a series of close-up snapshots of the contact area. As mentioned above, the crystals make contact after about $210 \mathrm{~ns}$, at which point only the edge of the upper crystal touches the upper surface of the bottom crystal. In the following $20 \mathrm{~ns}$, the water molecules move away from this contact line, inducing direct contact between the two cellulose-crystal surfaces. This direct contact leads to the formation of hydrogen bonds between the two cellulose-crystal surfaces, as shown in Fig. 4c. From 210 to $240 \mathrm{~ns}$, the number of hydrogen bonds rapidly increases as water is removed from the contact area; beyond $240 \mathrm{~ns}$, this number increases slowly, indicating a further extension of the contact area. These hydrogen bonds likely contribute to further stabilizing the contact between the two cellulose crystals. 
228 between the two cellulose crystals, despite the two surfaces in the contact being hydrophilic.

229 Previous atomistic MD simulations of similar duration indicate that water molecules are

230 often incorporated into aggregates of cellulose crystals in the aqueous condition (Chen et al.,

231 2019; Paajanen et al., 2019). The asymmetric geometry at the drying front induces

232 compressive stress at the extremity where the crystals touch each other, which would also

233 contribute to squeezing out water rather than evaporative drying. This removal of water

234 molecules from between the cellulose crystals and the subsequent tight adhesion of the

235 cellulose surfaces is presumably the main factor on the nanometric scale that causes the

236 collapse of the cellulose structure upon drying.

(a)

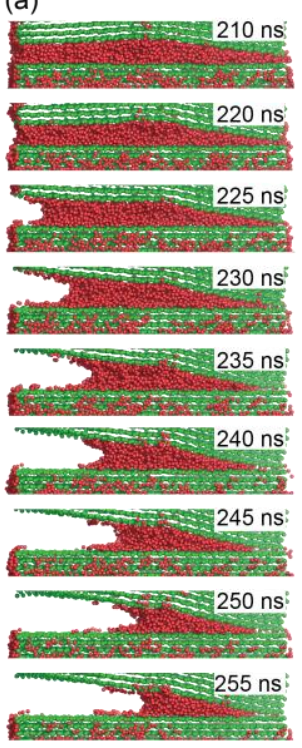

(b)
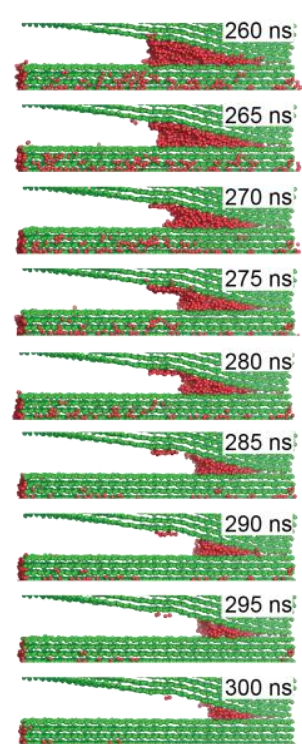
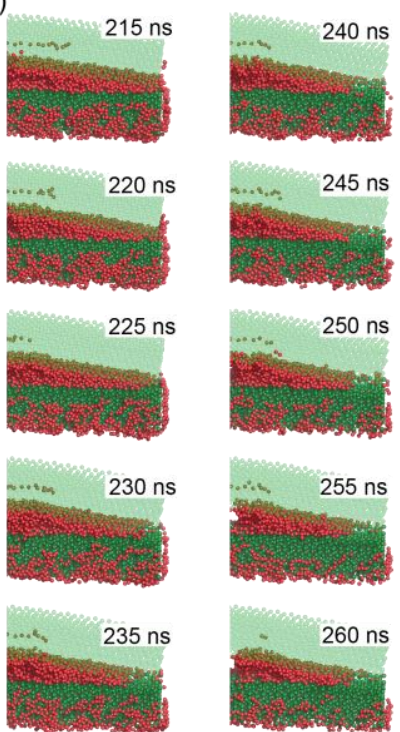

(c)

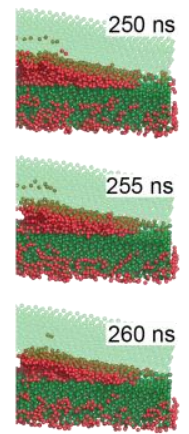

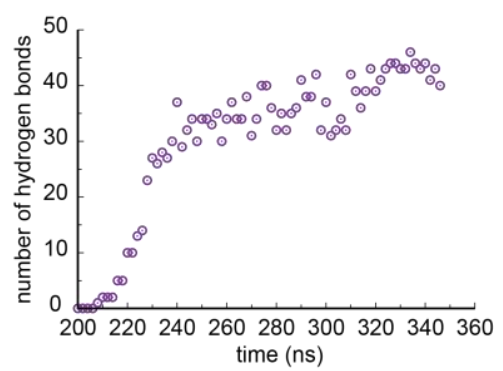

238 Figure 4. (a) Formation of water meniscus between two cellulose crystals. (b) Removal of water from the contact area between the two crystals. The upper crystal is translucent to reveal the water molecules between the crystals. (c) Number of hydrogen bonds between

241 the two cellulose-crystal surfaces as a function of time. 
By using atomistic MD simulations of a simplified cellulose-crystal system in water,

we show that drying may cause a significant bending deformation of a cellulose crystal and a tight adhesion between cellulose-crystal surfaces, even without external forces. The simulation shows that the crystal bending occurs not only because of the adhesion between cellulose crystals but also because of the surface tension of the surrounding water. The formation of a concave water meniscus in the opening between the two crystals is indicative of strong interactions between cellulose and water, and the water molecules are quickly excluded from the contact area between the two crystals. Such preferential drying between two crystals leads to adhesion between the cellulose surfaces.

The deformation of the upper cellulose crystal constitutes a complex phenomenon induced by the interplay between the cellulose crystals and water molecules, and the details of the simulated deformation may depend not only on the force-field parameters but also on the simulation conditions. Thus, the evolution of the deformation should be compared and verified with experimental observations such as atomic force microscopy images of deformed cellulose crystals. Such a comparison should allow a more complete understanding of the nanoscale structural modifications that are at the origin of the property changes of cellulosic materials.

\section{Acknowledgment}

We acknowledge the computational resources of the Centre d'Expérimentaion et de Calcul Intensif (CECIC) at the Institut de Chimie Moléculaire de Grenoble (ICMG) of the University Grenoble Alpes.

\section{References}


Abraham MJ, Murtola T, Shulz R, Páll S, Smith JC, Hess B, Lindahl E (2015) GROMACS: high performance molecular simulations through multi-level parallelism from laptops to supercomputers. SoftwareX 1-2:19-25

\section{1}

Beaumont M, König J, Opietnik M, Potthast A, Rosenau T (2017) Drying of a cellulose II gel: effect of physical modification and redispersibility in water. Cellulose 24:1199-1209

Berendsen HJC, Postma JPM, van Gunsteren WF, Hermans J (1981) Interaction models for water in relation to protein hydration. In Intermolecular Forces; Pullman, B., Ed.; dynamics with coupling to an external bath. J Chem Phys 81:3684-3690.

Bussi G, Donadio D, Parrinello M (2007) Canonical sampling through velocity rescaling. J Chem Phys 126:014101/1-014101/7

Chen P, Nishiyama Y, Mazeau K (2014) Atomic partial charges and one Lennard-Jones parameter crucial to model cellulose allomorphs. Cellulose 21:2207-2217

Chen P, Ogawa Y, Nishiyama Y, Ismail AE, Mazeau K (2016) Linear, non-linear and plastic bending deformation of cellulose nanocrystals. Phys Chem Chem Phys 18: 1988019887

Chen $P$, Ogawa $Y$, Nishiyama $Y$, Ismail AE, Mazeau $K$ (2018) l $\alpha$ to I $\beta$ mechano-conversion and amorphization in native cellulose simulated by crystal bending. Cellulose 25:43454355

Chen P, Terrenzi C, Furó I, Berglund LA, Wohlert J (2019) Quantifying localized macromolecular dynamics within hydrated cellulose fibril aggregates. Macromolecules 52: $7278-7288$ 
Fukuzumi H, Saito T, Iwata T, Kumamoto Y, Isogai A (2009) Transparent and high gas barrier

292

293

294

295

296

297

298

299

300

301

302

303

304

305

306

307

308

309

310

311 films of cellulose nanofibers prepared by TEMPO mediated oxidation. Biomacromolecules 10:162-165

Giacomozzi DE, Joutsimo O (2017) Drying temperature and hornification of industrial neverdried Pinus radiata Pulps. 2. Voith Sulzer Refining. BioResources 12:1532-1547

Häggkvist M, Li TQ, Ödberg, L (1998) Effects of drying and pressing on the pore structure in the cellulose fibre wall studied by ${ }^{1} \mathrm{H}$ and ${ }^{2} \mathrm{H}$ NMR relaxation. Cellulose 5:33-49

Hansen HS, Huenenberger PH (2011) A re-optimized GROMOS force field for hexopyranosebased carbohydrates accounting for the relative free energies of ring conformers, anomers, epimers, hydroxymethyl rotamers, and glycosidic linkage conformers. J Comput Chem 32:998-1032

Heath L, Thielemans W (2010) Cellulose nanowhisker aerogels. Green Chem 12:1448-1453

Hess B, Bekker H, Berendsen HJC, Fraaije JGEM (1997) LINCS: a linear constraint solver for molecular simulations. J Comput Chem 18:1463-1472

Hoepfner S, Ratka L, Milow B (2008) Synthesis and characterization of nanofibrillar cellulose aerogels. Cellulose 15:121-129

Hult EL, Larsson PT, Iversen T (2001) Cellulose fibril aggregation - an inherent property of kraft pulps. Polymer 42: 3309-3314

Jin H, Nishiyama Y, Wada M, Kuga S (2004) Nanofibrillar cellulose aerogels. Colloids Surf A

$$
\text { 240:63-67 }
$$

Lovikka VA, Khanjani P, Väisänen S, Vuorinen T, Maloney TC (2016). Porosity of wood pulp fibers in the wet and highly open dry state. Microporous Mesoporous Mater 234:326335. 
Mattos BD, Tardy BL, Rojas OJ (2019) Accounting for substrate interactions in the measurement of the dimensions of cellulose nanofibrils. Biomacromolecules 20:26572665

Minor JL (1994) Hornification - Its origin and meaning. Progress in paper recycling 3:93-95

Molnár G, Rodney D, Martoïa F, Dumont PJJ, Nishiyama Y, Mazeau K, Orgéas L (2018) Cellulose crystals plastify by localized shear. Proc Natl Acad Sci U.S.A. 115:7260-7265

Newman RH (2004) Carbon-13 NMR evidence for cocrystallization of cellulose as a mechanism for hornification of bleached kraft pulp. Cellulose 11: 45-52

Nishiyama Y, Langan P, Chanzy H (2002) Crystal structure and hydrogen-bonding system in cellulose $I \beta$ from synchrotron X-ray and neutron fiber diffraction. J Am Chem Soc $124: 9074-9082$

Ogawa, Y (2019) Electron microdiffraction reveals the nanoscale twist geometry of cellulose nanocrystals. Nanoscale 11:21767-21774

Oksanen T, Buchert J, Viikari L (1997) The role of hemicelluloses in the hornification of bleached kraft pulps. Holzforschung 51:355-360

Paajanen A, Ceccherini S, Maloney T, Ketoja JA (2019) Chirality and bound water in the hierarchical cellulose structure. Cellulose 26:5877-5892

Park S, Venditti RA, Jameel H, Pawlak JJ (2006) Changes in pore size distribution during the drying of cellulose fibers as measured by differential scanning calorimetry. Carbohydr Polym 66:97-103.

Peng Y, Gardner DJ, Han Y (2012) Dryomg cellulose nanofibrils: in search of a suitable method. Cellulose 19:91-102

PyMOL, The PyMOL Molecular Graphics System, Version 2.1.0 Schrödinger, LCC. 
337 Rämänen P, Penttilä P, Svedström K, Maunu SL, Serimaa R (2012) The effect of drying

338 method on the properties and nanoscale structure of cellulose whiskers. Cellulose

$339 \quad 19: 901-912$

340 Smitåh KB, Tisserant J-N, Assenza S, Arcari M, Nyström G, Mezzenga R (2019) Confinement-

341 induced ordering and self-folding of cellulose nanofibrils. Adv Sci 6:1801540.

342

DOI:10.1002/advs.201801540 